\title{
ÉTUDE DE LA SURVIE EMBRYONNAIRE APRES INDUCTION DE SUPEROVULATION CHEZ LA VACHE
}

\author{
J. TESTART, M.-J. BOSC et F. DU MESNIL DU BUISSON \\ avec la collaboration technique d'Anne-Marie MÉlières, Marie-José Prat \\ Y. de Fontaubert et $F$. Aymer de la Chevalerie \\ Institut national de la Recherche agronomique \\ Laboratoire de Physiologie de la Reproduction, 37-Nouzilly, France \\ Station centrale de Physiologie animale, 78 -Jouy-en-Josas, France
}

Chez les Bovins, la mortalité prénatale se manifeste tout au long de la gestation et on distingue la mortalité fœetale et la mortalité embryonnaire. Cette dernière survient au début de la gestation et entraîne généralement le retour en œestrus de la vache après régression du corps jaune. Très importante, la mortalité embryonnaire atteint I5 à $2 \mathrm{I}$ p. IOO des animaux, en conditions normales d'élevage, ainsi que le rapporte HANLY (I96I) et elle survient principalement avant le $26^{\mathbf{e}}$ jour de la gestation comme l'ont montré HAwk et al. (I955) sur des vaches de faible fertilité.

Par contre, après 40 jours de gestation, on n'observe que $8 \mathrm{p}$. roo environ de retours en cestrus après insémination (Couro'T, I968; Fosgate et Smith, I954) ce qui laisse supposer que la survie des embryons est principalement affectée au début de la gestation.

Après induction de superovulation chez la Vache, une mortalité se manifeste par des retours en chaleurs normaux et par des avortements (GoRdon et al., I962; HAFEZ et al., I965; MARTINENKo, I968). Une relation semble d'ailleurs avoir été observée entre le nombre d'œufs pondus et cette mortalité (GoRDON et al., I962; HAFEZ et al., I965). Nous avons donc été amenés à étudier la survie embryonnaire après superovulation chez la Vache.

\section{I. - MATÉRIEL E'T MÉTHODES}

Cent treize vaches de race Frisonne ont été réparties en trois lots. Quarante-huit vaches dont I I génisses, 27 primipares et ro multipares ont été soumises à la séquence suivante de traitement. PMSG-HCG : 2400 UI de PMSG (Roussel) ont été injectées par voie intramusculaire, 4 jours 
avant la date présumée de l'cestrus, et I 500 UL d'HCG (Roussel) par voie intraveineuse le jour de l'œstrus induit. Vingt-neuf vaches ont été saillies à cet œstrus et constituent le lot $\mathrm{r}$, les I9 autres ont été inséminées artificiellement avec du sperme congelé et forment le lot 2.

Soixante-cinq vaches primipares ont été soumises à un traitement combiné progestagènePMSG ; $200 \mathrm{mg}$ d'acétate de fluorogestone (SC-9 880) leur ont été administrés par voie vaginale au moyen d'éponges. Ces éponges placées le i $5^{\mathrm{e}}$ jour du cycle ( \pm I j) ont été maintenues en place pendant $2 \mathrm{I}$ jours. Une injection intramusculaire de PMSG (2 400 UI. Roussel ou I 600 UI Organon, équivalence établie d'après l'activité FSH dosée par la méthode de STEELman et PoHLEy) a été faite 2 à 5 jours avant le retrait de l'éponge. Ces vaches ont toutes été saillies à l'oestrus induit.

Le tableau I rapporte les taux de vaches gestantes en fonction du moment d'injection de PMSG par rapport à la fin du traitement progestatif.

\section{TABLEAU I}

Taux de vaches gestantes et multigestantes à $40 j$ de gestation, après induction de superovulation par traitement FGA-PMSG (lot 3)

\begin{tabular}{|c|c|c|c|c|c|}
\hline \multirow{2}{*}{$\begin{array}{c}\text { Injection PMSG } \\
\text { Retrait de l'éponge } \\
\text { vaginale }\end{array}$} & \multicolumn{4}{|c|}{ Intervalle (jours) } & \multirow{2}{*}{ Total } \\
\hline & -2 & -3 & -4 & -5 & \\
\hline Vaches traitées & 19 & 17 & 1' & 15 & 65 \\
\hline $\begin{array}{c}\text { P. } 100 \text { de vaches } \\
\text { gestantes }\end{array}$ & $\begin{array}{c}42,1 \\
(8)\end{array}$ & $\begin{array}{c}41,1 \\
(7)\end{array}$ & $\begin{array}{c}42,8 \\
(6)\end{array}$ & $\begin{array}{l}60,0 \\
(9)\end{array}$ & $\begin{array}{l}46,2 \\
(30)\end{array}$ \\
\hline $\begin{array}{l}\text { P. } 100 \text { de vaches } \\
\text { multigestantes }\end{array}$ & $\begin{array}{c}31,5 \\
(6)\end{array}$ & $\begin{array}{c}11,7 \\
(2)\end{array}$ & $\begin{array}{c}35,7 \\
(5)\end{array}$ & $\begin{array}{l}16,6 \\
(7)\end{array}$ & $\begin{array}{l}30,7 \\
(20)\end{array}$ \\
\hline
\end{tabular}

( ) nombre de vaches.

Le pourcentage de vaches gestantes étant semblable $(\mathrm{P}>0,05)$ quel que soit le moment d'injection de PMSG par rapport à la fin du traitement progestatif, nous avons regroupé toutes les vaches traitées en un seul lot (lot 3 ).

Après saillie ou insémination artificielle, toutes les vaches ont été soumises à un contrôle d'œstrus biquotidien. Les vaches non gestantes ont été abattues à leur premier retour en chaleur, les vaches gestantes entre le quarantième et le soixantième jour de la gestation.

A l'abattage, le poids des ovaires, le nombre et le poids des corps jaunes ont été notés de même que le nombre des embryons, leur poids, leurs mensurations et leurs battements cardiaques (test de survie). Lorsque ces derniers ont été retrouvés morts, la date approximative de leur mort a été estimée par comparaison de leur état, de leur poids et leurs mensurations aux données de Winters et al. (I953), de Fournier (I963), d'Hafez et Raj AKoski (I966).

\section{II. - RÉSULTATS ET DISCUSSION}

Dans le tableat 2, les résultats obtenus sont présentés de la façon suivante : dans chaque lot, nous avons réparti les animaux en deux classes : ceux dont les ovaires avaient I, 2, ou 3 corps jaunes et ceux dont les ovaires possédaient 4 corps jaunes et plus.

\section{Taux de gestation}

86,2 p. Ioo des vaches du lot I ont été gestantes alors qu'il n'y en eut que 57,8 p. Ioo dans le lot $2(\mathrm{P}<0,05)$ et 46,2 p. Ioo dans le lot $3(\mathrm{P}<0,05)$. Pour une même 
technique de superovulation, le mode d'insémination a donc une influence sur la fertilité des animaux, la saillie (lot I) étant supérieure à l'insémination artificielle (lot 2 ). Il faut reconnaître que les inséminations artificielles ont été effectuées dans de mauvaises conditions, ce qui explique en partie la faible proportion de vaches gestantes dans le lot 2. De même, les deux techniques de superovulation n'ont pas la même efficacité (lots I et 3) ; la fertilité est particulièrement faible après traitement progestatif : cela a déjà été observé par de nombreux auteurs sur vaches non superovulées.

TABLEAU 2

Survie embryonnaire à 40 jours de gestation, après induction de superovulation chez la vache

\begin{tabular}{|c|c|c|c|c|c|c|}
\hline \multirow[b]{2}{*}{ Lot } & \multirow{2}{*}{$\begin{array}{l}\text { C.J. par } \\
\text { vache }\end{array}$} & \multirow{2}{*}{$\begin{array}{l}\text { Nombre } \\
\text { de vaches }\end{array}$} & \multirow{2}{*}{$\begin{array}{l}\text { P. } 100 \text { de } \\
\text { vaches } \\
\text { gestantes }\end{array}$} & \multirow{2}{*}{$\begin{array}{c}\text { Nombre } \\
\text { moyen } \\
\text { de C.J. } \\
\text { par vache }\end{array}$} & \multicolumn{2}{|c|}{ P. 100 d'embryons } \\
\hline & & & & & $\begin{array}{l}\text { vivants } \\
\grave{a} 20 \mathrm{j}\end{array}$ & $\begin{array}{l}\text { morts entre } \\
20 \text { et } 40 \mathrm{j}\end{array}$ \\
\hline 1 & $\begin{array}{l}1-2-3 \\
\geqslant 4 \\
\text { total }\end{array}$ & $\begin{array}{r}20 \\
9 \\
29\end{array}$ & $\begin{array}{rr}85,0 & (17) \\
88,8 & (8) \\
86,2 & (25)\end{array}$ & $\begin{array}{l}1,47 \\
5,22 \\
2,68\end{array}$ & $\begin{array}{ll}67,7 & (21) \\
59,6 & (28) \\
62,8 & (49)\end{array}$ & $\begin{array}{rr}0,0 & (0) \\
25,0 & (7) \\
11,2 & (7)\end{array}$ \\
\hline 2 & $\begin{array}{l}1-2-3 \\
\geqslant 4 \\
\text { total }\end{array}$ & $\begin{array}{r}12 \\
7 \\
19\end{array}$ & $\begin{array}{rr}58,3 \quad(7) \\
57,1 \quad(4) \\
57,8 \quad(11)\end{array}$ & $\begin{array}{l}1,50 \\
5,00 \\
2,78\end{array}$ & $\begin{array}{ll}61,1 & (11) \\
31,2 & (12) \\
43,3 & (23)\end{array}$ & $\begin{array}{rr}0,0 & (0) \\
41,7 & (5) \\
21,7 & (5)\end{array}$ \\
\hline 3 & $\begin{array}{l}1-2-3 \\
\geqslant 4 \\
\text { total }\end{array}$ & $\begin{array}{l}43 \\
29 \\
65\end{array}$ & $\begin{array}{ll}46,5 & (20) \\
45,4 & (10) \\
46,2 & (30)\end{array}$ & $\begin{array}{l}1,79 \\
6,77 \\
3,47\end{array}$ & $\begin{array}{ll}38,9 & (30) \\
32,8 & (49) \\
34,9 & (79)\end{array}$ & $\begin{array}{rr}3, \ell_{1} & (1) \\
36,8 & (18) \\
24,0 & (19)\end{array}$ \\
\hline
\end{tabular}

Lot 1 : $\mathrm{PMSG}-\mathrm{HCG}+$ Saillie.

Lot 2: PMSG - HCG + Insémination artificielle.

Lot 3: Progestagène (FGA) - PMSG + Saillie.

( ) Nombre d'animaux.

De plus, pour chaque lot, le niveau d'ovulation n'a pas d'influence $(\mathbf{P}>0,05)$ sur les taux de vaches gestantes. D'autre part, les taux de vaches multigestantes ont été respectivement de 34,4 p. Ioo (lot I) ; 3I,5 p. Ioo (lot 2) et 30,7 p. Ioo (lot 3) ; ils sont indépendants $(\mathrm{P}>0,05)$ des techniques utilisées dans cette expérience.

\section{Taux de survie embryonnaire}

Les techniques de superovuiation utilisées ont des effets diffétents sur la survie embryonnaire. En effet, si l'on suppose que le nombre d'œufs pondus est égal à celui des corps jaunes, le taux de survie au $20^{\mathbf{e}}$ jour de gestation, exprimé par le rapport du nombre des embryons vivants à celui des corps jaunes, est plus élevé $(\mathrm{P}<0,05)$ dans le lot I que dans les lots 2 et 3 . Après superovulation modérée, ce taux, égal à 67,7 p. Ioo chez les vaches du lot $\mathrm{I}$, ne diffère pas de celui du lot 2 , égal à $6 \mathrm{I}$, I p. Ioo $(\mathrm{P}>0,05)$; par contre, il diffère de celui du lot 3 qui n'atteint que 38,9 p. Ioo $(\mathrm{P}<0,05)$. 
Après superovulation élevée, il est très différent entre les lots I et 3 (P<o,or), ceci souligne l'effet néfaste du traitement progestatif utilisé dans cette expérience.

Dans les expériences effectuées ici, la fécondabilité des œufs obtenus s'est révélée bonne. Ainsi, $75 \mathrm{p}$. Ioo environ des œufs ont été retrouvés après perfusion; 87 p. Ioo d'entre eux étaient fécondés. Lorsque la superovulation est limitée, ce qui se produit généralement pour les faibles doses de PMSG injectées, le taux de fécondation est de 90 à 95 p. Ioo, c'est-à-dire pratiquement identique à celui obtenu avec des œufs normaux. De même, après traitement par le progestagène Sc 9880 Searle on obtient un taux de fécondation acceptable bien que certains œufs ne semblent pas parfaitement normaux du point de vue structural (THIBAUL' $\mathbf{T}$, communication personnelle). Parmi les nombreux facteurs impliqués pour permettre un développement normal des œufs fécondés, on peut supposer que les conditions de passage dans l'oviducte et d'accueil dans l'utérus varient énormément d'un animal à l'autre suivant l'effet de l'induction de superovulation sur les ovaires. En effet, on peut penser que chez la Vache, comme chez la Brebis (WInTEnBERGER-Torres, I967), ces conditions de transit et d'accueil sont sous contrôle des stéroïdes ovariens.

Dans cette expérience, la mort des blastocystes est survenue très tôt, leur disparition complète entraînant le retour en chaleurs. Par contre, la disparition de certains d'entre eux, n'a pas apparemment affecté le développement des autres et le maintien de la gestation. En effet, lorsqu'un embryon meurt après 25 jours, on le retrouve à l'abattage 20 à 40 jours plus tard, avec ses annexes placentaires.

C'est ainsi qu'entre 20 et 40 jours de gestation, le nombre d'embryons morts est pratiquement nul, pour une superovulation modérée; pour une superovulation élevée, on observe un taux de mortalité important, variant suivant les lots de 25,0 p. Ioo à 4I,7 p. Ioo. Cette mortalité embryonnaire tardive est caractéristique de la vache superovulée. En effet, sur des vaches de faible fertilité, non superovulées (HAwk et al., I955), la mortalité embryonnaire est plus précoce et survient surtout avant le $26^{\mathbf{e}}$ jour de gestation.

\section{Effet du peuplement utérin}

I a mortalité tardive est liée au nombre d'embryons présents (tab1. 3). L'augmentation du nombre d'embryons soit par corne utérine, soit par utérus entraine celle du nombre d'embryons retrouvés morts après 40 jours de gestation, pour les vaches gestantes des trois lots. Bien que les classes de résultats soient différentes, il est intéressant de voir que cet effet de superovulation se manifeste, après 40 jours de gestation, de façon analogue et par corne utérine et par utérus.

Cet effet de surpopulation utérine a déjà été rapporté chez la Vache (GoRDON et al., I962 ; HAFEZ et al., I965). GORDON et ses collaborateurs remarquent qu'à 6 semaines de gestation, le nombre de fœetus survivants est lié au nombre d'ovulations. Ils concluent avec d'autres auteurs (HAFEz et al., I965; MARTINENKo, I965) que la possibilité à nourrir plus de deux foetus est limitée pour une corne utérine. Il est frappant de noter que cette mortalité tardive survient au moment de la mise en place de la placentation définitive, c'est-à-dire de la formation des placentomes (MELTON et al., I95I).

En conclusion, la mortalité embryonnaire se manifeste au cours de deux périodes après superovulation chez la Vache. La première est précoce ; après saillie et PMSG- 
HCG, elle atteint 37,2 p. Ioo des blastocystes et elle est beaucoup plus importante avec emploi de progestagène car elle affecte alors 65,I p. Ioo des blastocystes.

La seconde période de mortalité embryonnaire est plus tardive. Ellle coinncide avec la formation des placentomes et est liée à l'existence d'une population embryonnaire trop importante dans l'utérus.

TABLEAU 3

Influence de l'importance de la population utérine sur la survie embryonnaire à 40 jours de gestation

\begin{tabular}{|c|c|c|c|}
\hline $\begin{array}{c}\text { Nombre d'embryons par corne } \\
\text { utérine } \ldots \ldots \ldots \ldots \ldots \ldots \ldots \\
\text { Nombre de cas } \ldots \ldots \ldots \ldots \ldots \\
\text { Nombre d'embryons morts... }\end{array}$ & $\begin{array}{c}1 \\
53 \\
3 \\
(5,6 \%)\end{array}$ & $\begin{array}{c}2 \\
23 \\
11 \\
(93,9 \%)\end{array}$ & $\begin{array}{c}\geqslant \quad 3 \\
13 \\
25 \\
(18,0 \%)\end{array}$ \\
\hline $\begin{array}{l}\text { Nombre d'embryons par utérus. } \\
\text { Nombre de cas } \ldots \ldots \ldots \ldots \ldots \ldots \\
\text { Nombre d'embryons morts.... }\end{array}$ & $\begin{array}{c}1 \\
31 \\
0 \\
(0,0 \%)\end{array}$ & $\begin{array}{c}2 \text { ou } 3 \\
24 \\
11 \\
(18,0 \%)\end{array}$ & $\begin{array}{c}\geqslant \quad 4 \\
10 \\
28 \\
(17,5 \%)\end{array}$ \\
\hline
\end{tabular}

( ) Pourcentage des embryons retrouvés morts par rapport au total des embryons présents.

\section{RÉSUMÉ}

Nous avons étudié la survie embryonnaire chez la Vache saillie, après superovulation réalisée suivant deux techniques : l'une utilisant PMSG + HCG, l'autre couplant l'injection de PMSG avec un traitement de synchronisation d'oestrus (FGA). Nous avons aussi comparé, pour le traitement PMSG + HCG, l'efficacité de l'insémination artificielle ou de la saillie pour l'établissement d'une gestation. Pour cela, Ir 3 vaches de race Frisonne Pie-Noire ont été réparties en trois lots; elles ont toutes été abattues entre le $40^{\mathrm{e}}$ et le $60^{\mathrm{e}}$ jour de gestation.

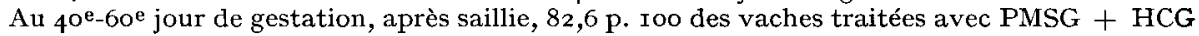
étaient gestantes contre 46,2 p. Ioo traitées avec FGA + PMSG. Après insémination artificielle, $57,8 \mathrm{p}$. Ioo seulement des vaches furent gestantes.

$\mathrm{Au} 2 \mathrm{O}^{\mathrm{e}}$ jour de gestation, et après saillie, le nombre d'embryons vivants ne représente que 34,9 p. Ioo des corps jaunes des vaches traitées avec FGA + PMSG; après traitement avec PMSG + HCG, ce nombre atteint 62,8 p. Ioo.

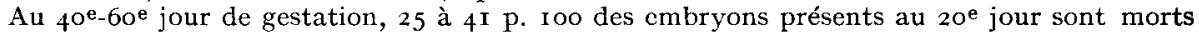
pour les vaches ayant 4 corps jaunes ou plus, alors que la survie embryonnaire n'est pas affectée pour les animaux ayant une superovulation modérée.

Ainsi peut-on distinguer, chez la Vache superovulée, deux périodes de mortalité embryonnaire ; la première est précoce, la seconde, plus tardive, est liéc au nombre d'embryons présents dans l'utérus au moment de la mise en place de la placentation définitive.

\section{SUMMARY}

\section{STUDY OF EMBRYONIC SURVIVAI, IN THE COW FOLLOWING INDUCED SUPEROVULATION}

Embryonic survival has been studied in cows mated after induced superovulation. 'Two techniques were used to induce ovulation :

-. One, applied during a normal œstrous cycle, involved an I. M. injection of I 600 I. U. 
of PMSG four days before the presumed date of ostrus and an I. V. injection of I 500 I. U. of $\mathrm{HCG}$ at the beginning of œstrus.

- The other one used an injection of I 600 I. U. of PMSG 2 at 5 days before the withdrawal of vaginal sponges impregnated with $200 \mathrm{mg}$ of fluorogestone acetate (FGA or Sc 988); that is, after 21 days of progestin treatment.

In the first case, artificial insemination or natural mating were used.

To that end, I 3 French Friesian cows were divided into three lots, and were slaughtered between days $40-60$ of pregnancy.

On days $40-60$ of pregnancy after natural mating, $82.6 \mathrm{p}$. Ioo of the cows treated with PMSG + HCG were pregnant as against 46.2 p. I00 in the FGA + IMMS group. After artificial insemination, only $57.8 \mathrm{p}$. Ioo were pregnant on day 20 of pregnancy. After natural mating the number of live embryos represents only $34.9 \mathrm{p}$. Ioo of the corpora lutea in cows treated with FGA + PMSG. After treatment with PMSG + HCG, this number reaches 62.8 p. Ioo.

On days 40-60 of pregnancy, 25-4I p. roo of the embryos present on day 20 were dead in cows having four or more corpora lutea, while embryonic survival was unaffected in the animals having a moderate superovulation.

Two periods of embryonic mortality may be distinguished in the supcrovulated cow: one is precocious; the second one occurs later, and is related to the number of enbryos present in the uterus at the time of the establishment of final placentation.

\section{RÉFÉRENCES BIBLIOGRAPHIQUES}

Courot M., Ig68. Communication personnelle.

Fosgate O. T., Smitu V. R., I954. Prenatal mortality in the bovine between pregnancy diagnosis at 34-50 days post insemination and parturition. J. Dairy. Sci., 37, ro7I-1073.

Focrnier C., I963. Dynamique morphologique au fatus bovin. Th. Doct. Vet. Alfort.

Gordon J., Wildiams G., EDWards J., I962. The use of PMS in the induction of twin pregnancy in the cow. J. Agric. Sci., 59, p. I43-198.

Hafez E. S. E., Jainudeen M. R., Lindsay D. A., I965, Cionadotropin induced twinning and related phenomena in beef cattle. Acta. Endocr., 50, suppl. Io2, 1-44.

Hafez E. S. E., Rajakoski E., I966. I'lacental and fetal development during multiple pregnancy. Anat. Rec., 150, 303-3I6.

Hanly S., i96r. Prenatal mortality in farm animals. J. Riprod. Fert, 2, 182-194.

HAwk et al., r955. Jimbryonic mortality between 16 and 34 days post breeding in cow of low fertility. J. Dairy. Sci., 38, 673-676.

Martinenko N. A., I 968 . Nortalité embryonnaire après stimulation ovarienne chez la Vache. VIe Congr. Reprod. Ins, Artif. Paris 1, 447-45o.

Melton A. A., Berry R. O., Butler O. D., I95 I. The interval between time of ovulation and attachment of the bovine embryo. J. Anim. Sici, 10, 995-1005.

Winters L. M., Green W. W., Comstok R. E., i953. Prenatal development of the bovine. Minnesota Techn. Bull., 151, pp. 43.

Wintenberger-Torres S., I967. Étude de la migration tubaire des aufs et de leur segmentation chez la Brebis. Thèse, Univ. Paris, no C. N. R. S; A.O. I 493. 